Neurol June 1995;37:810-814). (Respond: Dr Mott, Department of Neurology, Children's National Medical Center, 111 Michigan Ave, NW, Washington, DC 20010).

COMMENT. Neurologic complications of bone marrow transplantation in children with leukemia are a common occurrence. These have included seizures, infections, and encephalopathies, but Parkinsonian symptoms associated with amphotericin B appear to be unique.

\title{
HEMORRHAGIC SHOCK AND INFANTILE ENCEPHALOPATHY
}

The clinical characteristics, treatment and possible causes of hemorrhagic shock and encephalopathy in infants are described and a 5month-old patient is reported from the Section of Neurology, The Children's Mercy Hospital, Kansas City, MO. The infant presented with fever and irritability. She developed respiratory distress, requiring endotracheal intubation, followed by cardiorespiratory arrest. Excessive bleeding from puncture sites was associated with a disseminated intravascular coagulopathy. Admission diagnosis was septic shock. Other complications of this encephalopathy are bloody diarrhea and hepatorenal failure. Treatment requires fluids and electrolytes, fresh frozen plasma, and vitamin $\mathrm{K}$. Hyperthermia appeared important in causation. (Chaves-Carballo E. Hemorrhagic shock and encephalopathy: a new neurologic syndrome in infants. Acta Neuropediatr 1995;1:178-184). (Reprints: Dr E Chaves-Carballo, Section of Neurology, Children's Mercy Hospital, 2401 Gillham Road, Kansas City, MO 64108).

COMMENT. The syndrome was first described in Great Britain in 1983 as cited by the author (Levin $\mathrm{M}$ et al. Lancet 1983;2:64-67). The differential diagnosis includes septic shock, toxic-shock syndrome, Reye syndrome, and hemolytic-uremic syndrome. Early aggressive therapy was recommended.

\section{BRAIN NEOPLASMS}

\section{BRAIN TUMORS AND INFANTILE SPASMS}

Two patients, aged 6 and 7 months, with brain tumors who presented with infantile spasms and hypsarrhythmia are reported from Sapporo and Asahikawa Medical Universities, Japan. One had a hypothalamic hamartoma and the other a oligoastrocytoma with calcification in the right temporal lobe. ACTH controlled spasms and EEG seizure discharges. (Asanuma H et al. Brain tumors associated with infantile spasms. Pediatr Neurol May 1995;12:361-364). (Respond: Dr Asanuma, Department of Pediatrics, Sapporo Medical University, School of Medicine, S1 W16, Cho-ku, Sapporo, 060, Japan).

COMMENT. The authors cite 9 additional reports in the literature of brain tumors associated with infantile spasms. Focal brain lesions may underly the origin of infantile spasms.

Brain tumor was not listed as a cause of infantile spasms in an epidemiological study involving 57 patients treated in Sweden for the period 1987-1991. (Sidenvall R, Eeg-Olofsson O. Epilepsia July 1995;36:572-574).

PINEAL AND EPENDYMAL CYSTS AND INFANTILE SPASMS

A 3-month-old infant with infantile spasms and hypsarrhythmia 